\title{
INDUCTION CHEMOTHERAPY BEFORE SURGERY FOR EARLY-STAGE LUNG CANCER: A NOVEL APPROACH
}

K. M. W. Pisters, MD

R. J. Ginsberg, MD

D. J. Giroux, MS

J. B. Putnam, Jr, MD

M. G. Kris, MD

D. H. Johnson, MD

J. R. Roberts, MD

J. Mault, MD

J. J. Crowley, $\mathrm{PhD}$

P. A. Bunn, Jr, MD

On behalf of the Bimodality Lung

Oncology Team (BLOT)*
Objective: This phase II trial assessed the feasibility, as measured by response rate, toxicity, resectability rate, and surgical morbidity and mortality rates, of perioperative paclitaxel and carboplatin chemotherapy in patients with early-stage non-small cell lung carcinoma. Methods: All patients required negative mediastinoscopy results and adequate medical parameters to undergo induction chemotherapy and an operation. Superior sulcus patients were excluded. Chemotherapy consisted of paclitaxel $225 \mathrm{mg} / \mathrm{m}^{2}$ over 3 hours and carboplatin (area under the curve = 6) every 21 days for 2 cycles preoperatively. Three postoperative cycles of chemotherapy were planned for patients undergoing complete resection. Results: Between June 1996 and July 1998, 94 patients were entered into the study. Sixty-five (69\%) were men, and the median age was 64 years (range, 34-79 years). After induction chemotherapy, 53 of 94 (56\%; $95 \%$ confidence interval, $46 \%-67 \%$ ) had a major objective response, 88 (94\%) underwent surgical exploration, and 81 (86\%; 95\% confidence interval, $78 \%-92 \%$ ) underwent complete resection. Reasons for not undergoing an operation included disease progression $(n=3)$, clinically unresectable status $(n=1)$, death $(n=1)$, and patient lost to follow-up $(n=1)$. Two postoperative deaths occurred. Six $(6 \% ; 95 \%$ confidence interval, $0 \%-13 \%$ ) pathologic complete responses were observed. Ninety $(96 \%)$ patients received the planned preoperative chemotherapy versus $45 \%$ receiving postoperative chemotherapy. No unexpected chemotherapy or surgical morbidity occurred. The 1-year survival is currently estimated at $85 \%$, and the median survival has not yet been reached. Conclusions: Induction chemotherapy with paclitaxel and carboplatin is feasible and produces a high response rate with acceptable morbidity and mortality rates in early-stage non-small cell lung carcinoma. A prospective randomized trial comparing 3 cycles of induction chemotherapy and surgery with surgery alone in early-stage non-small cell lung carcinoma is planned. (J Thorac Cardiovasc Surg 2000;119:429-39)
I 1999 , lung cancer will be diagnosed in an estimated 171,600 patients. $^{1}$ Of these new cases, approximately $80 \%$ will have non-small cell lung cancer (NSCLC). Although the most common presentation is metastatic disease, many patients present with potentially operable disease. Despite surgical resection, patients with clinical

From the Bimodality Lung Oncology Team (BLOT).

Supported in part by a grant from Bristol-Myers Squibb. All authors had research/grant support from Bristol-Myers Squibb for conduct of this trial. Drs Pisters, Ginsberg, Mault, and Bunn receive honoraria from Bristol-Myers Squibb for educational lectures.

Read at the Seventy-ninth Annual Meeting of The American Association for Thoracic Surgery, New Orleans, La, April 18-21, 1999.

Received for publication April 22, 1999; revisions requested July 12, 1999; revisions received Oct 15, 1999; accepted for publication Oct 25,1999 stage T2 N0, T1-2 N1, and T3 N0-1 NSCLC have poor 5 -year survivals $(38 \%, 34 \%, 24 \%, 22 \%$, and $9 \%$, respectively). ${ }^{2}$ Although the disease is presumed to be confined to the chest, it recurs in the majority of patients undergoing resection, and they die of metastases. Trials evaluating postoperative radiotherapy, chemotherapy, or

\footnotetext{
Address for reprints: Katherine M. W. Pisters, MD, Department of Thoracic/Head \& Neck Medical Oncology, U.T. M. D. Anderson Cancer Center, 1515 Holcombe Blvd, Box 80, Houston, TX 77030-4095 (E-mail address: kpisters@mdanderson.org).

*For listing of BLOT participating institutions and co-investigators, see end of article.

Copyright @ 2000 by Mosby, Inc.

0022-5223/2000 $\$ 12.00+0 \quad \mathbf{1 2 / 6 / 1 0 4 1 5 7}$
}

doi:10.1067/mtc. 2000.104157 
both in resectable NSCLC have been disappointing. In contrast, randomized trials have demonstrated improved survivals for patients with locally advanced disease treated with cisplatin-based induction chemotherapy before radiation therapy ${ }^{3,4}$ or surgery. ${ }^{5,6}$ Given the survival statistics and failure of postoperative treatment to substantially improve outcome for patients with earlystage resectable NSCLC, this trial was designed to explore perioperative chemotherapy in patients with clinical stage IB (T2 N0), II (T1-2 N1, T3 N0), and selected IIIA (T3 N1) disease extent.

The chemotherapy regimen of paclitaxel and carboplatin has been extensively studied in cases of metastatic NSCLC. ${ }^{7-13}$ This combination has been found to be well tolerated. The most severe toxicities are neutropenia and peripheral neuropathy, which are observed in the minority of patients who generally receive 3 to 4 cycles. The data from all of these studies suggest that the combination of paclitaxel and carboplatin is active. We elected to use a 3-hour paclitaxel schedule because it can be given on an outpatient basis without routine growth factor support. The response rates with 225 $\mathrm{mg} / \mathrm{m}^{2}$ given over 3 hours appear equivalent to those observed with the more myelosuppressive 24-hour infusions by using lower doses.

Two cycles of preoperative chemotherapy were planned because most responses in metastatic disease had been observed after 2 cycles of chemotherapy ${ }^{7-13}$ and to avoid excessive delay before surgery in this early-stage group of patients. Three cycles of postoperative chemotherapy were incorporated into the study design because the randomized trial by Roth and colleagues ${ }^{5}$ for stage III disease had used both preoperative and postoperative chemotherapy, and the majority of the planning committee recommended additional chemotherapy for patients experiencing major tumor regression after the 2 preoperative cycles.

The main objective of this study was to establish the feasibility, as measured by the response rate and toxicity of perioperative chemotherapy, resectability rate, pathologic complete response (CR) rate, and surgical mortality rate, of a perioperative regimen of paclitaxel and carboplatin in patients with early-stage NSCLC. A secondary objective was to prospectively estimate the survival of patients with NSCLC treated with this approach, with a view toward a randomized trial comparing perioperative chemotherapy plus surgery with surgery alone.

\section{Methods}

From June 1996 to July 1998, 94 patients with histologically confirmed NSCLC were entered into this multicenter trial. All patients had clinical stage T2 N0, T1-2 N1, or T3 N0-1 NSCLC, as defined by history, physical examination, routine laboratory evaluation, chest computed tomography (CT) scan, bronchoscopy, and mediastinoscopy. Clinical T stage was defined by CT imaging. Clinical nodal stage was assessed by mediastinoscopy and CT imaging. A negative biopsy result of all enlarged $(>1.0 \mathrm{~cm}$ on CT scan) mediastinal lymph nodes was required. Clinically involved N1 nodes (level 10 hilar, 11, 12, and 13) were defined as having a short diameter greater than $1.0 \mathrm{~cm}$ on CT scan confirmed by a radiologist. Patients with superior sulcus tumors were excluded because of a competing study. Patients were required to have no prior chemotherapy or radiation therapy, be 18 years or older, have Zubrod performance status of 0 to 1 , and have adequate hematologic, renal, and hepatic function to safely undergo chemotherapy and surgery. Patients were also required to have a predicted postresection forced expiratory volume in 1 second of $1.0 \mathrm{~L}$ or more and bidimensionally measurable disease or evaluable indicator lesions. Informed consent was obtained from all patients, and the protocol was reviewed and approved by the institutional review board of each participating institution.

Pretreatment evaluation included a CT scan of the chest and upper abdomen (to include the adrenal glands), posteroanterior and lateral chest $\mathrm{x}$-ray films, electrocardiograms, pulmonary function tests (spirometry, arterial blood gas, and diffusing capacity), bronchoscopy, and mediastinoscopy in addition to routine laboratory tests. Imaging studies of the brain and bone were required only if clinically indicated. All toxicities were graded according to Southwest Oncology Group toxicity criteria.

\section{Treatment plan}

Preoperative chemotherapy. Eligible patients received 2 cycles of preoperative chemotherapy with paclitaxel (225 $\mathrm{mg} / \mathrm{m}^{2}$ as a 3-hour intravenous infusion) and carboplatin (area under the curve of 6, as determined by the Calvert formula; administered intravenously over 30 minutes after the paclitaxel infusion) every 21 days. Before the second cycle of chemotherapy, patients had repeat laboratory tests and a chest $\mathrm{x}$-ray film. Patients with evidence of disease progression after 1 or 2 cycles of preoperative chemotherapy were removed from protocol treatment and recommended for surgical exploration if appropriate. All patients were premedicated for chemotherapy with a combination of dexamethasone, diphenhydramine, and an $\mathrm{H}_{2}$ antagonist according to standard practice. No dose escalation was allowed. Subsequent doses of chemotherapy were modified for toxicity if appropriate. A major objective response after induction chemotherapy consisted of complete response (CR) or partial response (PR). $\mathrm{CR}$ was defined as total resolution of all radiographic findings after chemotherapy, and PR was recorded when the sum of the products of perpendicular diameters of all measurable lesions was reduced by $50 \%$ or more from baseline. Progressive disease was defined as a $50 \%$ increase in the product of the tumor diameters or appearance of any new lesion. All other patients were considered to have stable disease.

Surgery. Patients without evidence of progressive disease 
Table I. Patient characteristics

\begin{tabular}{lc}
\hline No. of patients & 94 \\
Median age, y (range) & $64(34-79)$ \\
Men (\%) & $65(69)$ \\
Women (\%) & $29(31)$ \\
Zubrod performance status (\%) & \\
0 & $50(53)$ \\
1 & $44(47)$ \\
Pretreatment clinical stage (\%) & \\
IB, T2 N0 & $42(45)$ \\
IIA, T1 N1 & $1(1)$ \\
IIB, T2 N1 & $27(29)$ \\
IIB, T3 N0 & $17(18)$ \\
IIIA, T3 N1 & $7(7)$ \\
Histology (\%) & \\
Squamous cell carcinoma & $41(44)$ \\
Adenocarcinoma & $32(34)$ \\
NSCLC, not otherwise specified & $16(17)$ \\
Large cell carcinoma & $5(5)$ \\
Elevated serum lactate dehydrogenase $(\%)^{*}$ & $12(13)$ \\
\hline
\end{tabular}

*Measurements for 3 patients were not available.

underwent thoracotomy within 3 to 6 weeks of their second administration of paclitaxel and carboplatin. A repeat staging evaluation 2 to 6 weeks after the second paclitaxel and carboplatin administration was performed and included the following: bronchoscopy (for patients with T3 N0 disease assessable only by bronchoscopy), repeat pulmonary function tests, electrocardiograms, chest x-ray films, and a CT scan of the chest and upper abdomen.

At thoracotomy, the extent of pulmonary resection was left to the discretion of the attending surgeon as long as the resection chosen provided complete removal of the primary lesion with negative gross and microscopic margins (R0). Segmentectomy and wedge resection were discouraged. All accessible hilar lymph nodes were dissected from the specimen, and a complete mediastinal lymph node dissection was performed in all patients. For right-sided lesions, this included 2R, 4R, 7, 8, 9, and 10R. For left-sided lesions, this included $4 \mathrm{~L}, 5,6,7,8,9$, and $10 \mathrm{~L}$.

Postoperative chemotherapy and follow-up. Patients who did not undergo complete resection (positive microscopic margins, R1; gross disease, R2) and were found to have diseased (positive) mediastinal nodes, T4 lesions, or metastatic disease at surgery were removed from protocol treatment. Patients who had unacceptable morbidity were removed from study treatment and received further therapy at the discretion of their physicians. All patients were followed up to ascertain recurrence, long-term toxicities, and survival. Patients who underwent complete resection and whose disease did not progress on induction chemotherapy were to receive 3 cycles of postoperative paclitaxel and carboplatin chemotherapy (same dose and schedule as preoperative cycles) beginning within 8 weeks of thoracotomy. After completion of protocol therapy, patients were followed up with an interval history, physical examinations, and chest x-ray films every 3 months for 1 year and then every 6 months thereafter.
Table II. Induction chemotherapy response

\begin{tabular}{lrrr}
\hline & No. & $\%$ & $95 \%$ CI \\
\hline CR & 1 & 1 & \\
PR & 52 & 55 & \\
Major response & 53 & 56 & $46-67$ \\
Stable disease & 32 & 34 & \\
Progression & 3 & 3 & \\
Not evaluable & $6^{*}$ & & \\
Total & 94 & & \\
\hline
\end{tabular}

*There were 2 paclitaxel reactions, 2 patients with inadequate data, 1 patient lost to follow-up, and 1 induction death.

Statistical considerations. The main objective of this trial was to establish the feasibility of perioperative paclitaxel and carboplatin treatment in patients with clinical stage IB, II, and selected IIIA NSCLC. The key end points were response rate and toxicities of chemotherapy, pathologic CR rate, resectability rate, and surgical morbidity and mortality rates. With an accrual of 80 patients, the resectability rate, response rate, and surgical mortality rate could be estimated to within $\pm 11 \%$ (approximate $95 \%$ confidence interval $[\mathrm{CI}]$ ). A preliminary assessment of the primary study end points occurred after the initial 20 eligible patients had undergone surgery and had been followed up for 1 month. No unexpected morbidity or mortality had occurred, and the protocol continued to enroll patients. Time to progression and survival were calculated as the elapsed time between the date of registration for study and the first documentation of recurrence or death, respectively. All patients will be followed up until death.

\section{Results}

Ninety-four patients were accrued, with a median age of 64 years. Sixty-five (69\%) patients were male (Table I). Patients had a Zubrod performance status of 0 or 1 . Pretreatment clinical disease stage was as follows: T2 N0, 42 patients; T1 N1, 1 patient; T2 N1, 27 patients, T3 N0, 17 patients, and T3 N1, 7 patients. Squamous cell carcinoma was the most frequent histologic subtype $(44 \%)$. Twelve $(13 \%)$ patients had an elevated serum lactate dehydrogenase level at study entry.

Induction chemotherapy. Ninety $(96 \%)$ patients received both cycles of paclitaxel and carboplatin preoperatively. Four patients did not complete induction treatment. Three patients were removed from the study because of allergic reactions to paclitaxel, and 1 patient died of a cerebrovascular accident after the first cycle of induction chemotherapy (see below).

Response to induction chemotherapy. The radiographic response to induction chemotherapy is presented in Table II. One patient had a clinical CR, and 52 patients had PRs. The overall major objective clinical 
Table III. Surgical results

\begin{tabular}{|c|c|c|c|c|c|}
\hline $\begin{array}{l}\text { Clinical } \\
\text { stage }\end{array}$ & $\begin{array}{c}\text { No. of } \\
\text { patients }\end{array}$ & $\begin{array}{c}\text { No } \\
\text { surgery }\end{array}$ & Explored & $R 0$ & $\begin{array}{l}\text { hologic } \\
C R\end{array}$ \\
\hline T2 N0 & 42 & $2^{*}$ & 40 & 38 & 3 \\
\hline T1 N1 & 1 & 0 & 1 & 1 & 1 \\
\hline $\mathrm{T} 2 \mathrm{~N} 1$ & 27 & $1^{\dagger}$ & 26 & 26 & 2 \\
\hline T3 N0 & 17 & $1^{\ddagger}$ & 16 & 12 & 0 \\
\hline T3 N1 & 7 & $2^{\S}$ & 5 & 4 & 0 \\
\hline Total (\%) & 94 & $6(6)$ & $88(94)$ & $81(86)$ & $6(6)$ \\
\hline $95 \%$ CI & & & & $78 \%-92 \%$ & $0 \%-13 \%$ \\
\hline
\end{tabular}

$R 0$, Complete resection.

"One patient had progression of disease, and there was 1 induction death.

'One patient had progression of disease.

One patient had clinically unresectable disease.

$\S$ One patient had progression of disease, and 1 patient was lost to follow-up.
Table IV. Surgical treatment

\begin{tabular}{|c|c|}
\hline Treatment & No. of patients \\
\hline Lobectomy & 68 \\
\hline Lobectomy & 58 \\
\hline Lobe plus chest wall & 7 \\
\hline Lobe plus sleeve & 3 \\
\hline Pneumonectomy & 13 \\
\hline Simple & 9 \\
\hline Pneumonectomy plus chest wall & 2 \\
\hline Intrapericardial pneumonectomy & 2 \\
\hline Bilobectomy & 4 \\
\hline Exploration only & 2 \\
\hline Wedge resection & 1 \\
\hline Total & 88 \\
\hline
\end{tabular}

Table V. Correlation of clinical and pathologic staging

\begin{tabular}{|c|c|c|c|c|}
\hline \multirow[b]{2}{*}{ Clinical stage } & \multirow[b]{2}{*}{ Explored } & \multicolumn{3}{|c|}{ Pathologic (surgical) stage } \\
\hline & & Less extensive & Equivalent & More extensive \\
\hline \multirow[t]{10}{*}{ T2 N0 $(n=42)$} & 40 & $12(30 \%)$ & $7(17 \%)$ & $21(53 \%)$ \\
\hline & & $\mathrm{TO} \mathrm{NO}=3$ & & $\mathrm{~T} 3 \mathrm{NO}=3$ \\
\hline & & $\mathrm{T} 1 \mathrm{~N} 0=9$ & & $\mathrm{~T} 4 \mathrm{NO}=1$ \\
\hline & & & & $\mathrm{T} 1 \mathrm{~N} 1=3$ \\
\hline & & & & $\mathrm{T} 2 \mathrm{~N} 1=5$ \\
\hline & & & & $\mathrm{T} 3 \mathrm{~N} 1=1$ \\
\hline & & & & $\mathrm{T} 4 \mathrm{~N} 1=1$ \\
\hline & & & & $\mathrm{T} 2 \mathrm{~N} 2=5$ \\
\hline & & & & $\mathrm{T} 2 \mathrm{~N} 0, \mathrm{~T} 1 \mathrm{~N} 0=1$ \\
\hline & & & & T3 N0 M1 = 1 \\
\hline \multirow[t]{2}{*}{ T1 N1 (n = 1) } & 1 & $1(100 \%)$ & & \\
\hline & & $\mathrm{T} 0 \mathrm{NO}=1$ & & \\
\hline \multirow[t]{6}{*}{$\mathrm{T} 2 \mathrm{~N} 1(\mathrm{n}=27)$} & 26 & $16(62 \%)$ & $4(15 \%)$ & $6(23 \%)$ \\
\hline & & $\mathrm{T} 0 \mathrm{NO}=2$ & & $\mathrm{~T} 3 \mathrm{~N} 1=1$ \\
\hline & & $\mathrm{T} 1 \mathrm{NO}=6$ & & $\mathrm{~T} 4 \mathrm{NO}=1$ \\
\hline & & $\mathrm{T} 2 \mathrm{~N} 0=6$ & & $\mathrm{~T} 2 \mathrm{~N} 2=3$ \\
\hline & & $\mathrm{T} 0 \mathrm{~N} 1=1$ & & $\mathrm{~T} 2 \mathrm{~N} 0, \mathrm{~T} 1 \mathrm{~N} 0=1$ \\
\hline & & $\mathrm{T} 1 \mathrm{~N} 1=1$ & & \\
\hline \multirow[t]{5}{*}{ T3 N0 $(n=17)$} & 16 & $8(50 \%)$ & $3(19 \%)$ & $5(31 \%)$ \\
\hline & & $\mathrm{T} 1 \mathrm{~N} 0=4$ & & $\mathrm{~T} 3 \mathrm{~N} 1=2$ \\
\hline & & $\mathrm{T} 2 \mathrm{NO}=3$ & & $\mathrm{~T} 3 \mathrm{~N} 2=1$ \\
\hline & & $\mathrm{T} 2 \mathrm{~N} 1=1$ & & $\mathrm{~T} 3 \mathrm{~N} 3=1$ \\
\hline & & & & $\mathrm{T} 4 \mathrm{~N} 1=1$ \\
\hline \multirow[t]{4}{*}{ T3 N1 (n = 7) } & 5 & $5(100 \%)$ & & \\
\hline & & $\mathrm{T} 1 \mathrm{~N} 0=1$ & & \\
\hline & & $\mathrm{T} 2 \mathrm{NO}=2$ & & \\
\hline & & $\mathrm{T} 3 \mathrm{NO}=2$ & & \\
\hline Total & 88 & $42(48 \%)$ & $14(16 \%)$ & $32(36 \%)$ \\
\hline
\end{tabular}

Less extensive, Pathologic stage less extensive than clinical stage; equivalent, pathologic stage the same as the clinical stage; more extensive, pathologic stage more extensive than clinical stage.

response rate after induction chemotherapy was $56 \%$ (95\% CI, 46\%-67\%). Stable disease was seen in 32 (34\%) patients, and disease progression was seen in 3 (3\%). Six patients were not evaluable for response to induction chemotherapy. Two had paclitaxel reactions during their first cycle of chemotherapy, 2 had inadequate imaging data, 1 was lost to follow-up, and 1 died.

Surgical treatment. After chemotherapy, 88 (94\%) of the 94 patients underwent surgical exploration. Reasons for not undergoing surgical exploration are 
Table VI. Pathologic staging versus clinical response

\begin{tabular}{|c|c|c|c|c|c|c|c|c|}
\hline \multirow[b]{3}{*}{ Clinical stage } & \multirow[b]{3}{*}{ No. of patients } & \multirow[b]{3}{*}{ Explored } & \multicolumn{6}{|c|}{ Pathologic stage } \\
\hline & & & \multicolumn{2}{|c|}{ Less } & \multicolumn{2}{|c|}{ Equivalent } & \multicolumn{2}{|c|}{ More } \\
\hline & & & $P R$ & $S D$ & $P R$ & $S D$ & $P R$ & $S D$ \\
\hline T2 N0 & 42 & 40 & 10 & 2 & 2 & $5^{*}$ & 10 & 11 \\
\hline T1 N1 & 1 & 1 & 1 & 0 & 0 & 0 & 0 & 0 \\
\hline $\mathrm{T} 2 \mathrm{~N} 1$ & 27 & 26 & $10^{\dagger}$ & 6 & 4 & 0 & 4 & 2 \\
\hline T3 N0 & 17 & 16 & 4 & $4^{\dagger}$ & 1 & 2 & 2 & $3^{\S}$ \\
\hline T3 N1 & 7 & 5 & 5 & 0 & 0 & 0 & 0 & 0 \\
\hline Total & $\overline{94}$ & $\overline{88}$ & $\overline{30}$ & $\overline{12}$ & 7 & $\overline{7}$ & $\overline{16}$ & $\overline{16}$ \\
\hline \multicolumn{3}{|c|}{ Major response rate } & \multicolumn{2}{|c|}{$30 / 42(71 \%)$} & \multicolumn{2}{|c|}{$7 / 14(50 \%)$} & \multicolumn{2}{|c|}{$16 / 32(50 \%)$} \\
\hline
\end{tabular}

$P R$, Partial response ( $\geq 50 \%$ decrease in the product of the tumor diameters); $S D$, stable disease

${ }^{*}$ One patient was not evaluable for clinical response (paclitaxel reaction).

$\dagger$ One patient had a clinical CR.

¥Two patients were not evaluable for clinical response (paclitaxel reaction).

${ }^{\S}$ Two patients were not evaluable for clinical response (inadequate data).

Table VII. Induction chemotherapy toxicity $(n=94)$

\begin{tabular}{|c|c|c|c|c|c|c|c|}
\hline \multirow[b]{2}{*}{ Toxicity } & \multicolumn{7}{|c|}{ Grade } \\
\hline & Unknown & 0 & 1 & 2 & 3 & 4 & 5 \\
\hline Allergy & 1 & 88 & 0 & 2 & 1 & 2 & 0 \\
\hline \multicolumn{8}{|l|}{ Hematologic } \\
\hline Anemia & 1 & 41 & 44 & 7 & 0 & 1 & 0 \\
\hline Granulocytopenia & 1 & 29 & 14 & 17 & 16 & 17 & 0 \\
\hline Thrombocytopenia & 1 & 83 & 9 & 0 & 1 & 0 & 0 \\
\hline Infection & 1 & 85 & 1 & 1 & 6 & 0 & 0 \\
\hline Malaise/fatigue/lethargy & 1 & 55 & 22 & 16 & 0 & - & - \\
\hline Myalgia/arthralgia & 1 & 38 & 26 & 24 & 5 & - & - \\
\hline Nausea & 1 & 68 & 15 & 9 & 1 & 0 & 0 \\
\hline \multicolumn{8}{|l|}{ Neuropathy } \\
\hline Numbness & 2 & 82 & 0 & 9 & 1 & 0 & 0 \\
\hline Paresthesia & 1 & 60 & 28 & 2 & 3 & 0 & 0 \\
\hline Phlebitis/thrombosis/embolism & 1 & 91 & 0 & 1 & 0 & 0 & 1 \\
\hline Vomiting & 1 & 79 & 7 & 6 & 1 & 0 & 0 \\
\hline
\end{tabular}

listed in Table III. Complete resection (R0) of all residual tumor at the primary site and in lymph nodes was possible in 81 patients $(86 \%$; $95 \%$ CI, $78 \%-92 \%)$. The types of resection included lobectomy in 68 , pneumonectomy in 13 , bilobectomy in 4 , exploration only in 2, and wedge resection in 1 patient (Table IV).

Surgical pathologic findings for each subset of clinically staged patients are presented in Table V. Pathologic CR observed in 6 patients $(6 \% ; 95 \%$ CI, 0\%-13\%) occurred only among major responders clinically. Overall, $48 \%$ of patients had a pathologic stage that was less extensive than their pretreatment clinical stage, $16 \%$ had an equivalent stage, and $36 \%$ had more extensive disease. Of the 42 patients whose pathologic stage was less than their clinical stage at protocol entry, $30(71 \%)$ had a radiographic major response to induction therapy (Table VI). Fifty percent of patients who were found to have equivalent or more extensive disease at surgery had major chemotherapy responses.

Postoperative treatment. Forty-three (46\%) patients did not receive postoperative chemotherapy. Twentyeight were considered to be "off study" because of surgical pathologic findings (19), no surgery (6), or prior paclitaxel reaction (3). Fifteen patients who were considered "on study" did not receive postoperative chemotherapy because of prolonged postoperative recovery/complications (6), poor performance status (2), postoperative death (2), neuropathy (1), elevated 
liver transaminase level (1), discovery of metastatic disease (1), being lost to follow-up (1), and inadequate data (1). Forty-two (45\%) patients received all 3 of the planned postoperative paclitaxel and carboplatin chemotherapy cycles. Eight $(8 \%)$ patients received only 2 cycles because of treatment-limiting fatigue (3), neuropathy (2), error (1), physician decision to begin radiotherapy (1), and an unknown cause (1). One patient received only 1 cycle because of treatment limiting neuropathy. Of the 66 patients potentially eligible to receive postoperative chemotherapy, 42 (64\%) received all 3 cycles.

Treatment-related complications. Table VII displays the significant toxicities observed during the 2 cycles of induction chemotherapy. Three patients experienced grade 3 or 4 paclitaxel-induced hypersensitivity reactions. Severe anemia or thrombocytopenia occurred in only 1 patient each. Grade 3 or 4 neutropenia occurred in $35 \%$ of patients; however, no patient was admitted with febrile neutropenia. Forty-one percent of patients complained of grade 1 or 2 fatigue, but none had grade 3 fatigue. Myalgias and arthralgias were common, with $26(28 \%)$ patients reporting grade 1 (mild), 24 (26\%) reporting grade 2 (decreased ability to move), and $5(5 \%)$ reporting grade 3 (disabling). This complication generally occurred several days after chemotherapy and resolved within 1 week. Nausea and vomiting were uncommon. Peripheral neuropathy of moderate severity was noted in 4 patients $(4 \%)$, and none had grade 4 neuropathy.

There was one death during induction chemotherapy. This occurred in a 71-year-old woman after recovery from chemotherapy-related toxicity. The patient was hospitalized after the first cycle of chemotherapy with grade 4 neutropenia, hyponatremia, and mental status changes. The patient had seizures and was believed to have had a cerebrovascular accident. After resolution of her neutropenia and hyponatremia, the patient was discharged to a nursing facility, where she died after extension of her cerebrovascular accident.

Table VIII displays the surgical morbidity data. No unusual toxicities were noted. In all patients pulmonary function tests were performed before protocol entry and again before surgery. There were no significant changes in these tests. There were 2 postoperative deaths. One occurred in a 61-year-old man with clinical T2 N1 disease. This patient experienced a PR after chemotherapy, underwent lobectomy with complete resection, and was found to have T2 N0 disease at surgery. On postoperative day 4 , the patient became febrile and had progressive shortness of breath necessitating intubation. The patient died after a prolonged stay in the intensive care unit. The second postoperative death occurred in a 69-year-old man with clinical T2 N0 disease. This patient had a PR to induction therapy and underwent complete resection with pneumonectomy but had T2 N2 disease at surgery. On postoperative day 1 , the patient became acutely short of breath requiring reintubation and died shortly thereafter. The presumed cause of death was a pulmonary embolus.

Postoperative chemotherapy toxicity data are displayed in Table IX. No additional or unexpected toxicities occurred.

Survival. Survival data are presented in Fig 1. The 1and 2-year survivals for the 94 patients were $85 \%$ and $56 \%$, respectively. The median survival has not yet been reached. Interpretation of this survival curve is hampered by the relative nonhomogeneity of the clini$\mathrm{cal} /$ pathologic stages of the patients treated.

\section{Discussion}

Patients with clinically staged T2 N0, T1 N1, T2 N1, and T3 N0-1 NSCLC have poor 5-year survival (38\%$9 \%$ ) when treated with surgery alone. ${ }^{2}$ Overall results with postoperative chemotherapy have found a $5 \%$ or less improvement in survival at 5 years. ${ }^{14}$ Postoperative radiotherapy has not been shown to improve survival. ${ }^{15}$

In the past decade numerous phase II trials have examined the use of induction chemotherapy (with or without radiotherapy) followed by surgery for stage III NSCLC. ${ }^{16-19}$ Induction therapy offers several potential advantages, including (1) early control of micrometastatic disease, (2) reduction in size of the primary tumor, (3) patient acceptance (vs postoperative therapy), and (4) patient compliance because of better tolerability. These single-institution trials found that preoperative chemotherapy was feasible and resulted in higher response rates than previously seen in patients with stage IV disease. In patients with stage III disease so treated, complete resection rates ranged from $50 \%$ to $75 \%$, and pathologic CRs (no viable tumor found in the resection specimen) were found in $5 \%$ to $15 \%$ of patients. Pathologic CR is predictive of long-term survival. ${ }^{20}$ Median survivals reported in these studies were comparable (18-24 months), with 5-year survivals from $15 \%$ to $25 \%$.

Two prospective randomized trials have compared primary surgery with induction chemotherapy and surgery in stage IIIA NSCLC. ${ }^{5,6}$ Both trials were terminated early after interim analyses indicated a survival advantage for the chemotherapy-treated patients. Cisplatin-based chemotherapy was administered preoperatively for 3 cycles in each trial, and an additional 
Table VIII. Surgical toxicity $(n=88)$

\begin{tabular}{lccccccc}
\hline & \multicolumn{5}{c}{ Grade } \\
\cline { 2 - 7 } Toxicity & Unknown & 0 & 1 & 2 & 3 & 4 & 5 \\
\hline Arrhythmia & 8 & 67 & 1 & 1 & 9 & 2 & 0 \\
Hemorrhage & 7 & 80 & 0 & 0 & 1 & 0 \\
Lung (other) & 13 & 56 & 2 & 5 & 10 & 1 & 0 \\
Phlebitis/thrombosis/embolism & 7 & 80 & 0 & 0 & 0 & 0 \\
Pneumonitis/effusions/infiltrates & 12 & 72 & 1 & 1 & 2 & 0 \\
Pulmonary edema & 7 & 79 & 0 & 0 & 2 & 0 & 0 \\
Respiratory infection & 7 & 70 & 0 & 1 & 10 & 0 \\
Wound infection & 7 & 80 & 0 & 0 & 1 & 0 \\
\hline
\end{tabular}

Table IX. Postoperative chemotherapy toxicity $(n=51)$

\begin{tabular}{|c|c|c|c|c|c|c|c|}
\hline \multirow[b]{2}{*}{ Toxicity } & \multicolumn{7}{|c|}{ Grade } \\
\hline & Unknown & 0 & 1 & 2 & 3 & 4 & 5 \\
\hline Allergy & 0 & 51 & 0 & 0 & 0 & 0 & 0 \\
\hline \multicolumn{8}{|l|}{ Hematologic } \\
\hline Anemia & 4 & 23 & 22 & 2 & 0 & 0 & 0 \\
\hline Granulocytopenia & 4 & 12 & 9 & 3 & 10 & 13 & 0 \\
\hline Thrombocytopenia & 4 & 31 & 13 & 3 & 0 & 0 & 0 \\
\hline Infection & 4 & 46 & 1 & 0 & 0 & 0 & 0 \\
\hline Malaise/fatigue/lethargy & 4 & 23 & 9 & 13 & 2 & - & - \\
\hline Myalgia/arthralgia & 4 & 29 & 5 & 11 & 2 & - & 一 \\
\hline Nausea & 4 & 37 & 5 & 4 & 1 & 0 & 0 \\
\hline \multicolumn{8}{|l|}{ Neuropathy } \\
\hline Numbness & 4 & 30 & 0 & 12 & 5 & 0 & 0 \\
\hline Paresthesia & 4 & 29 & 14 & 3 & 1 & 0 & 0 \\
\hline Phlebitis/thrombosis/embolism & 4 & 47 & 0 & 0 & 0 & 0 & 0 \\
\hline Vomiting & 4 & 42 & 1 & 2 & 2 & 0 & 0 \\
\hline
\end{tabular}

3 cycles of chemotherapy were given postoperatively to the patients in the M. D. Anderson study. Postoperative irradiation was given to all patients in the Spanish trial and only to patients having incomplete resection in the M. D. Anderson trial. Response to induction chemotherapy was $60 \%$ in the Barcelona study and $35 \%$ in the M.D. Anderson study. Both trials showed a significant improvement in survival for patients treated with induction chemotherapy. This survival advantage persisted with multivariate analysis and long-term follow-up.

On the basis of the success of induction chemotherapy before surgery in stage III NSCLC, we and others have designed studies to evaluate the role of induction chemotherapy before surgery in earlier-stage NSCLC. Depierre and colleagues ${ }^{21}$ from France have reported preliminary results of a randomized trial of induction chemotherapy with mitomycin, ifosfamide, and cisplatin plus surgery compared with surgery alone in stages IB, II, and IIIA NSCLC. A total of 373 patients were entered into the study, and 355 were eligible. One hundred eighty-eight had stage I or II disease, whereas 167 had stage IIIA disease. Pathologic CRs occurred in $19(11 \%)$, and pathologic downstaging occurred in a further $95(54 \%)$ of the patients treated with chemotherapy. A 10-month improvement in the median survival (26 vs 36 months, $P=.11$ ) was seen. Postoperative morbidity was more common in the combined modality arm, with an increased rate of fistula and empyema observed. The postoperative mortality rate was also increased in the combined modality arm, but this was not statistically significant. Survival was then separately examined within the first 150 days and thereafter because of nonproportional hazards over 


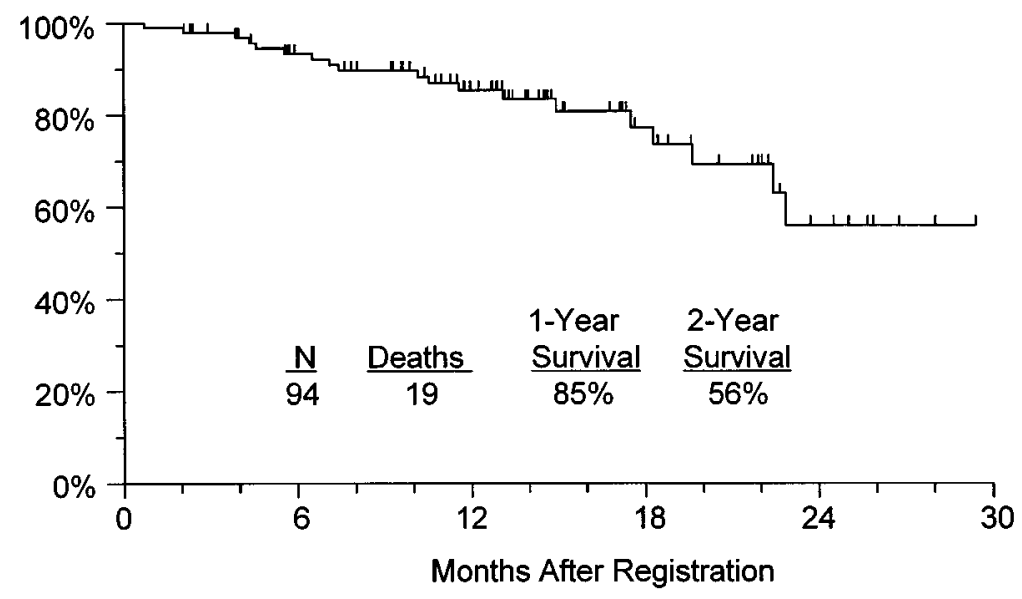

Fig 1. Overall survival curve.

time in treatment groups. After a nonsignificant excess of deaths in the combined modality arm during the treatment period, the effect of combined modality therapy after 150 days was significantly favorable for survival. No difference was seen in local recurrence rate; however, a significant decrease in distant metastases was seen favoring the chemotherapy arm. Disease-free survival was significantly longer in the chemotherapy arm $(P=.02)$. The authors concluded that the use of combined modality therapy resulted in a significant decrease in the risk of death for patients with earlystage NSCLC, and a significant survival benefit was seen beyond 5 months.

The clinical response rate of $56 \%$ seen in this multiinstitutional trial may seem low in comparison with the phase II experience of cisplatin-based chemotherapy in stage III disease. However, this response rate is more than double the $22 \%$ response rate seen in the multiinstitutional trial comparing paclitaxel and carboplatin (same dose and schedule) to etoposide and cisplatin in patients with inoperable disease. ${ }^{22}$ The pathologic CR rate of $6 \%$ is lower than that seen for cisplatin-based trials in patients with stage III NSCLC. However, many patients had evidence of pathologic downstaging but did not fulfill the criteria for complete histologic sterilization. The randomized trial comparing induction paclitaxel and carboplatin with surgery alone will include 3 cycles of preoperative chemotherapy, which we hope will improve the major response and pathologic CR rates.

This trial also serves to emphasize that despite modern CT imaging and mediastinoscopy, clinical staging was inaccurate, with only $16 \%$ of patients having an equivalent clinical and pathologic stage and $36 \%$ hav- ing more extensive disease at surgery (Table V). Some of this inaccuracy may have been due to a positive downstaging effect of the chemotherapy. The routine use of positron emission tomography (PET) scanning may improve the diagnostic accuracy of clinical staging in the future. Use of PET scanning will be encouraged but not required for the planned phase III trial.

Ninety-six percent of the enrolled patients received the induction chemotherapy as planned. In contrast, only $43(46 \%)$ of the patients enrolled received the planned 3 postoperative cycles of chemotherapy. Of the 66 patients who were potentially eligible to receive the postoperative chemotherapy (ie, not off study for pathologic/surgical stage, progression on induction chemotherapy, or paclitaxel allergic reaction), only 42 (64\%) received the full 3 cycles. This experience is similar to that seen in other postoperative adjuvant trials conducted in stage III NSCLC using high-dose cisplatin chemotherapy, ${ }^{23-25}$ during which patients were often unable to receive the entire prescribed dose.

This multi-institutional phase II trial has demonstrated that induction chemotherapy with paclitaxel and carboplatin is feasible and safe in early-stage NSCLC. Response rates and pathologic findings suggest promise for this approach in improving survival in early-stage resectable NSCLC. Induction chemotherapy before surgery may provide the best approach for using combined modality therapy in the management of NSCLC.

A phase III North American intergroup trial comparing primary surgery alone with induction chemotherapy with paclitaxel and carboplatin followed by surgery is planned. This national trial is anticipated to open in 1999. 


\section{BLOT Participating Institutions and Co-Investigators}

K. M. W. Pisters, J. B. Putnam, Jr, J. C. Nesbitt, and J. S. Lee, M. D. Anderson Cancer Center, Houston, Texas; R. J. Ginsberg, R. T. Heelan, M. G. Kris, and V. Rusch, Memorial Sloan-Kettering Cancer Center, New York, New York; D. H. Johnson and J. R. Roberts, Vanderbilt University, Nashville, Tennessee; P. A. Bunn, Jr, W. Franklin, and J. Mault, University of Colorado Cancer Center, Denver, Colorado; R. Cameron and R. A. Figlin, University of California, Los Angeles, California; F. Detterbeck and M. A. Socinski, University of North Carolina, Chapel Hill, North Carolina; L. Robinson and J. C. Ruckdeschel, H. Lee Moffitt Cancer Center, Tampa, Florida; D. J. Giroux and J. J. Crowley, Cancer Research and Biostatistics, Seattle, Washington.

We acknowledge Kristie Beasley from Cancer Research and Biostatistics and the research nurses and data managers at each participating institution for their assistance in the conduct of this trial.

\section{REFERENCES}

1. Landis SH, Murray T, Bolden S, Wingo PA. Cancer Statistics, 1999. CA Cancer J Clin 1999;49:8-31.

2. Mountain CF. Revisions in the International System for Staging Lung Cancer. Chest 1997;111:1710-7.

3. Dillman RD, Seagren SL, Propert KF, et al. A randomized trial of induction chemotherapy plus high-dose radiation versus radiation alone in stage III non-small cell lung cancer. N Engl J Med 1990; 323:940-5.

4. Sause WT, Scott C, Taylor S, et al. Radiation therapy oncology group (RTOG) and Eastern Cooperative Oncology Group (ECOG) 4588: preliminary results of a phase III trial in regionally advanced, unresectable non-small cell lung cancer. J Natl Cancer Inst 1995;87:198-205.

5. Roth JA, Fossella F, Komaki R, et al. A randomized trial comparing perioperative chemotherapy and surgery with surgery alone in resectable stage IIIA non-small cell lung cancer. J Natl Cancer Inst 1994;86:673-680.

6. Rosell R, Gomez-Codina J, Camps C, et al. A randomized trial comparing preoperative chemotherapy plus surgery with surgery alone in patients with non-small cell lung cancer. N Engl J Med 1994;330:153-8.

7. Langer CJ, Leighton JC, Comis RL, et al. Paclitaxel by 24-hour or 1-hour infusion in combination with carboplatin in advanced non-small cell lung cancer: the Fox Chase Cancer Center experience. Semin Oncol 1995;22(Suppl 9):18-29.

8. Belani CP, Hiponia D, Engstrom C, et al. Phase I study of paclitaxel and carboplatin in advanced and metastatic non-small cell lung cancer [abstract]. Proc Am Soc Clin Oncol 1995;14:A381.

9. Johnson DH, Paul DM, Hande KR, et al. Paclitaxel (taxol) plus carboplatin for advanced lung cancer: preliminary results of a Vanderbilt University phase II trial-LUN-46. Semin Oncol 1995; 22(Suppl 9):30-3.

10. Muggia FM, Vafai D, Natale R, et al. Paclitaxel 3-hour infusion given alone and combined with carboplatin: preliminary results of dose-escalation trials. Semin Oncol 1995;22(Suppl 9):63-6.

11. Rowinsky EK, Flood WA, Sartorius SE, et al. Phase I study of paclitaxel as a 3-hour infusion followed by carboplatin in untreat- ed patients with stage IV non-small cell lung cancer. Semin Oncol 1995;22(Suppl 9):48-54.

12. Bunn PA, Kelly K. A phase I study of carboplatin and paclitaxel (taxol) in non-small cell lung cancer: a University of Colorado Cancer Center study. Semin Oncol 1995;22(Suppl 9):2-6.

13. Giaccone G, Huizing M, Postmus P, et al. Dose-finding and sequencing study of paclitaxel and carboplatin in non-small cell lung cancer. Semin Oncol 1995;22(Suppl 9):78-82.

14. Non-Small Cell Lung Cancer Collaborative Group. Chemotherapy in non-small cell lung cancer: a meta-analysis using updated data on individual patients from 52 randomized trials. BMJ 1995;311:899-909.

15. PORT Meta-Analysis Trialists Group. Postoperative radiotherapy in non-small cell lung cancer: systematic review and meta-analysis of individual patient data from nine randomized controlled trials. Lancet 1998;352:257-63.

16. Burkes RL, Ginsberg RJ, Shepherd FA, et al. Induction chemotherapy with mitomycin, vindesine, and cisplatin for stage III unresectable non-small cell lung cancer: results of the Toronto phase II trial. J Clin Oncol 1992;10:580-6.

17. Darwish S, Minotti V, Crino L, et al. Neoadjuvant cisplatin and etoposide for stage IIIA (clinical N2) non-small cell lung cancer. Am J Clin Oncol 1994;17:64-7.

18. Martini N, Kris MG, Flehinger BJ, et al. Preoperative chemotherapy for stage IIIA (N2) lung cancer: the Memorial SloanKettering experience with 136 patients. Ann Thorac Surg 1993; 55:1365-74.

19. Vokes EE, Bitran JD, Hoffman PC, et al. Neoadjuvant vindesine, etoposide, and cisplatin for locally advanced non-small cell lung cancer. Chest 1989;96:110-3.

20. Pisters KMW, Kris MG, Gralla RJ, et al. Pathologic complete response in advanced non-small cell lung cancer following preoperative chemotherapy: implications for the design of future non-small cell lung cancer combined modality trials. J Clin Oncol 1993;11:1757-62.

21. Depierre A, Milleron B, Moro D, et al. Phase III trial of neo-adjuvant chemotherapy in resectable stage I (except T1 N0), II, and IIIa non-small cell lung cancer: the French experience [abstract]. Proc Am Soc Clin Oncol 1999;18:465a.

22. Belani CP, Natale RB, Lee JS, et al. Randomized phase III trial comparing cisplatin/etoposide versus carboplatin/paclitaxel in advanced and metastatic non-small cell lung cancer [abstract]. Proc Am Soc Clin Oncol 1998;17:455a.

23. Feld R, Rubinstein L, Thomas PR, the Lung Cancer Study Group. Adjuvant chemotherapy with cyclophosphamide, doxorubicin, and cisplatin in patients with completely resected stage I non-small cell lung cancer. J Natl Cancer Inst 1993;85:299-306.

24. The Lung Cancer Study Group. Final analysis. Chest 1994;106: 279S-410S.

25. Pisters KMW, Kris MG, Gralla RJ, et al. Randomized trial comparing postoperative chemotherapy with vindesine and cisplatin plus thoracic irradiation with irradiation alone in stage III (N2) non-small cell lung cancer. J Surg Oncol 1994;56:236-41.

\section{Discussion}

Dr Mark K. Ferguson (Chicago, Ill). Dr Pisters, you and your co-authors have performed an important clinical trial aimed at determining the potential benefit of neoadjuvant chemotherapy in early-stage NSCLC. As surgeons, our inability to achieve adequate cure rates with the single- 
modality therapy of resection, even in early-stage lung cancer, has been evident for decades. The reluctance of the surgical and medical communities to investigate multimodality therapy has been based on the relatively low efficacy of traditional multiagent regimens and the high toxicity associated with them. New agents and combinations permit outpatient therapy with relatively low toxicity, have improved patient acceptance, and demonstrate good clinical response rates, thus providing the opportunity for studies such as this.

Your report is a valuable one, demonstrating that a trial of neoadjuvant chemotherapy for early-stage lung cancer can accrue patients successfully and produces relatively good clinical response rates with low morbidity. These are the results the authors predicted but form an important basis for future work. The results also raise some interesting questions.

First, is this the right subset of patients to select for neoadjuvant therapy? In particular, why do you omit patients with stage IA disease, whose survival is only marginally better than that of patients with stage IB disease? Also, why include patients with $\mathrm{T} 3$ disease who had an $\mathrm{R} 0$ resection rate of only $60 \%$ and who accounted for a large fraction of the patients taken off the protocol? Would it not be best to concentrate on patients with $\mathrm{T} 1$ or $\mathrm{T} 2$ or N0 or N1 disease so as not to muddy the waters?

Second, in the presentation and in the article constant reference is made to clinical stage rather than pathologic stage. Unfortunately, even with routine mediastinoscopy, clinical staging in this trial was less than $20 \%$ accurate, with at least $35 \%$ of patients being understaged. Did you consider using a more sensitive clinical staging algorithm that included such things as PET scanning or thoracoscopy?

Finally, I have concern regarding the complete pathologic response rate of only $5 \%$ for this protocol. We know from other trials in lung cancer and esophageal cancer in which the response rates vary from $20 \%$ to $30 \%$ that only those patients who have a pathologic CR have a survival advantage over the rest of the group. With such a low CR rate in this trial, what are the implications of this protocol therapy regarding survival? Is it appropriate to begin a randomized trial having demonstrated such a poor complete clinical response rate? If such a trial is instituted, how many patients will have to be entered to achieve the statistical power needed to answer this important question?

Dr Pisters. To answer your first question about whether this is the right subset, we excluded patients with stage IA disease when a group of medical oncologists and thoracic surgeons met to originally plan the trial. We did that because the surgeons in the crowd believed that was a very favorable subset and they did not want to subject them to the rigors of chemotherapy before surgery. I would be quite happy to treat them, but I think I might have problems finding those patients.

We included patients with T3 N0 disease because we decided to include patients with early-stage lung cancer up to the $\mathrm{N} 2$ classification. We believed that the literature had demonstrated a survival benefit for combined modality therapy in patients with N2 disease, but it was less clear for patients with earlier stage disease, and that is why the patients with T3 N0 and $\mathrm{N} 1$ disease were entered.

In terms of the clinical and pathologic staging, that is a point that we tried to emphasize in the article. This decision is made based on clinical staging, and there is such a difference in survivals between patients who have clinical staging and surgical staging. Furthermore, as our experience has demonstrated, clearly many of the patients who we believed had early-stage disease had a pathologic stage that was really much more advanced.

In the randomized trial it has been proposed that PET scanning will be used as a companion study both at the onset of clinical staging and again before surgery to look at whether PET scanning accurately predicts clinical response and pathologic response. We did not address the use of thoracoscopy for staging. I would defer on that issue to my surgical colleagues.

I was disappointed that the pathologic CR rate was only $5 \%$. There were many other patients who had significant pathologic responses seen that did not qualify as pathologic CRs in which $75 \%$ or $80 \%$ of the resected specimen was necrotic. I agree that pathologic CR is a very important predictor for long-term survival, especially in patients who have only received chemotherapy before surgery as opposed to patients who have had chemotherapy and radiation before surgery, where I think that sterilization of the tumor after radiation does not have that same translation to long-term survival and control.

In the randomized trial we will be using 3 cycles of chemotherapy before surgery, hoping that we will convert a few more patients from histologic downstaging to complete sterilization, and I believe that the statistical section calls for approximately 600 patients to enter that randomized trial.

Dr David J. Sugarbaker (Boston, Mass). Dr Pisters, you mentioned the clinical identification of $\mathrm{N} 1$ disease. In the absence of PET scanning, how did you determine that patients did indeed have N1 disease?

Dr Pisters. To qualify for the trial, all patients underwent a mediastinoscopy. Therefore, even if they had CT evidence of mediastinal lymph node enlargement, if their mediastinoscopy result was negative, we determined that they were N2 negative. N1 involvement was determined by reviewing the $\mathrm{CT}$ scan and the mediastinal windows and was defined as the presence of bronchopulmonary or hilar lymph nodes that were greater than $1 \mathrm{~cm}$.

Dr Sugarbaker. Because we know that enlarged mediastinal nodes on CT scan will be benign at the time of mediastinoscopy roughly $40 \%$ to $50 \%$ of the time, I think most of us would expect that the same would hold true for N1 adenopathy. Therefore I am wondering whether that might be a confounding element here.

Dr Pisters. I agree, but I do not know how we can address that other than by doing what we did. I guess the use of PET scanning may be helpful and will be done in the randomized trial.

Dr Sugarbaker. I have one further follow-up question. With such a dismal CR rate, have you considered adding radiotherapy up front? 
Dr Pisters. I think that the use of radiation before a definitive resection just makes it harder for the surgeon to remove the tumor. I am not sure that radiation, which is another form of local control, is really going to help when most of these patients die of systemic recurrence and metastases.

Dr Tom K. Waddell (Toronto, Ontario, Canada). Given phase I and phase II trials demonstrating no increased efficacy of the chemotherapy regimen used in this study compared with cisplatin-based regimens, can you comment on the relative cost of your chemotherapy regimen?

Dr Pisters. When this trial was designed, it fell on the heels of an American Society of Clinical Oncology meeting in which there had been a lot of phase II data presenting the combination of paclitaxel and carboplatin that appeared very promising, and some of those trials were multicenter trials. There was heated discussion among the medical oncologists present about what the optimum chemotherapy would be.
Subsequent phase III trials comparing paclitaxel and carboplatin to cisplatin-based regimens have really demonstrated equivalence in terms of both response and survival. Therefore there is no clear winner, and there is no clear loser. Many people have adopted paclitaxel and carboplatin, at least in the United States, as their front-line therapy for NSCLC because it can be administered once every 3 weeks and is generally well tolerated. Cost is an issue, and it is interesting that as time goes on, the other pharmaceutical companies are raising the cost of their medicines to be equivalent to what people are paying for the paclitaxel and carboplatin.

Dr Scott J. Swanson (Boston, Mass). Do you have any information available regarding the reason why 8 patients underwent incomplete resection in this early-stage group?

Dr Pisters. In general, the reason was diseased margins, but I do not have specific data on all 8 patients.

\section{Bound volumes available to subscribers}

Bound volumes of The Journal of Thoracic and Cardiovascular Surgery are available to subscribers (only) for the 2000 issues from the Publisher, at a cost of $\$ 134.00$ for domestic, $\$ 165.85$ for Canadian, and $\$ 155.00$ for international subscribers for Vol 119 (January-June) and Vol 120 (July-December). Shipping charges are included. Each bound volume contains a subject and author index and all advertising is removed. Copies are shipped within 60 days after publication of the last issue of the volume. The binding is durable buckram with the Journal name, volume number, and year stamped in gold on the spine. Payment must accompany all orders. Contact Mosby, Inc, Subscription Services, 11830 Westline Industrial Drive, St Louis, MO 63146-3318, USA; phone 800-453-4351 or 314-453-4351.

Subscriptions must be in force to qualify. Bound volumes are not available in place of a regular Journal subscription. 